\title{
Indicadores para medir la calidad de servicio y normas de protocolo y etiqueta, aplicando el modelo servqual en el restaurante Tapas \& Topes, de Puyo, Provincia de
} Pastaza

Indicators to measure the quality of service and rules of etiquette and protocol, applying the servqual model in the restaurant Tapas \& Topes, of

$$
\text { Puyo, Province of Pastaza }
$$

Tania Cristina Cevallos Punguil. ${ }^{1}$, María Belén Bastidas Arauz. ${ }^{2}$ Andrea Estefanía Fierro Ricaurte. ${ }^{3} \&$ Elsa Ordóñez Bravo ${ }^{4}$.

\begin{abstract}
DOI: $\underline{\text { https://doi.org/10.33262/cienciadigital.v3i1.263 }}$

The objective of this work is to measure the quality of the service and protocol and etiquette standards at the Tapas \& Topes restaurant in the city of Puyo, province of Pastaza, understanding that well-focused service and attention can be an excellent strategy market to position the service offered in the mind of the guest and build loyalty with the service. For this purpose, the Servqual tool was used, which measures the quality of the service, through the expectations and perceptions of the clients, on five dimensions: reliability, sensitivity, security, empathy, and tangible elements.

It was found that $6.2 \%$ said they had received an excellent service, $59.37 \%$ received a very good service, $28.1 \%$ received a good service and $6.25 \%$ a service of poor quality. In general, there is a significant value that is considered good service. In the evaluation of the standards of label and protocol, the checklist was applied, resulting in $41 \%$ of the personnel having little knowledge and needing to be trained in this subject. The contribution that this research provides is mainly focused on the perception and

\footnotetext{
${ }^{1}$ Universidad Estatal Amazónica. Observatorio Turístico de la Universidad Estatal Amazónica. Ecuador. tc.cevallosp@uea.edu.ec

${ }^{2}$ Escuela Superior Politécnica del Chimborazo. Docente de la Carrera de Gastronomía. Ecuador m_bastidas@ espoch.edu.ec

3 Escuela Superior Politécnica del Chimborazo. Docente de la Carrera de Gastronomía. Ecuador. andrea.fierro@espoch.edu.ec

4 Escuela Superior Politécnica del Chimborazo. Docente de la Carrera de Gastronomía. Ecuador. elsa.ordoñez@espoch.edu.ec
} 
expectation of the client about the service, which allows the restaurant TAPAS \& TOPES to make decisions based on the results offered by the application of this tool.

Keywords: Quality indicators. Protocol and Label. Servqual model.

\section{Resumen}

El presente trabajo tiene por objetivo medir la calidad del servicio y normas de protocolo y etiqueta, en el restaurante Tapas \& Topes, de la ciudad de Puyo, provincia de Pastaza, entendiendo así que el servicio y atención bien enfocados pueden ser una excelente estrategia de mercado para posicionar el servicio ofrecido en la mente del huésped y fidelizarlo con el servicio. Para este fin se utilizó la herramienta Servqual, que mide la calidad del servicio, mediante las expectativas y percepciones de los clientes, sobre cinco dimensiones: fiabilidad, sensibilidad, seguridad, empatía, y elementos tangibles.

Se encontró que un 6,2\% manifestaron haber recibido un excelente servicio, un $59,37 \%$ recibió un muy buen servicio, el 28,1\% recibió un servicio bueno y el 6,25\% un servicio de calidad deficiente. En general se tiene un valor significativo que es considerado como buen servicio. En la evaluación sobre las normas de etiqueta y protocolo se aplicó el check list, dando como resultado que el $41 \%$ del personal tiene poco conocimiento y requiere ser capacitado en este tema. El aporte que brinda esta investigación se centra fundamentalmente en la percepción y expectativa del cliente sobre el servicio, lo que permite al restaurante TAPAS \& TOPES tomar decisiones en base a los resultados que ofrece la aplicación de esta herramienta.

Palabras Claves: Indicadores de calidad del servicio, Protocolo y Etiqueta, Modelo Servqual

\section{Introducción.}

La calidad del servicio es un factor imprescindible que debe existir en la empresa para tener un mayor número de clientes y clientes satisfechos. Según Menéndez y Motto (2014) enfatizaron que la calidad del servicio es una parte fundamental en el proceso de venta del establecimiento. Todos los departamentos existen, precisamente, para atraer clientela, vender y rentabilizar el establecimiento. Pero el contacto directo con el cliente, lo tiene el personal de servicios, que debe vender lo que otros han hecho, empleando sus habilidades y conocimientos.

Desde otra perspectiva, Cantú. H, (2011) considera que la calidad del servicio, es el conjunto de valores y hábitos que posee una persona, que complementados con el uso de prácticas y herramientas de calidad en el actuar diario, le permiten colaborar con su organización para afrontar los retos que se le presenten, en el cumplimiento de la misión de la organización. Para que una empresa alcance el éxito y más si esta es de turismo que, al ser una actividad 
sensible por el trato directo que tiene con el cliente, debe tener presente lo manifestado por Castro y Moros (2015), quienes enfatizan sobre la importancia de las organizaciones (de bienes y servicios) quienes deben tomar la calidad como una opción a seguir y en la que todas las personas involucradas en el proceso productivo deben participar, no como una imposición de la dirección.

La creciente globalización de los mercados ha incrementado considerablemente la competitividad entre los distintos sectores económicos. Y la industria restaurantera no está exenta a estos cambios, cada vez son más innovadores con mayores propuestas hacia los clientes con variedad de comida y diferentes sabores. La competencia por captar mayor número de clientes ha motivado a muchos restaurantes a mejorar la calidad de servicio porque el mercado cada vez se pone más exigente, pero hay restaurantes que no se esfuerzan en brindar al cliente un buen servicio.

En la actualidad existe en el mercado una alta competencia gastronómica y con ello un aumento en la exigencia de la calidad de servicio. Los clientes piden un trato individualizado y por esta razón las empresas deben centrar sus esfuerzos en lograr la satisfacción de los clientes, que para Vera \& Trujillo (2009), está muy relacionada con la calidad del servicio. Feijó (2016), señala que la finalidad de la calidad de servicio se entiende como el nivel de excelencia que un establecimiento se ha marcado con la intención de satisfacer las expectativas del cliente, es un sistema del que participa toda la organización y que permite descubrir las causas que producen defectos, con el fin de reducir los costos y ganar en satisfacción del cliente. Este concepto de calidad no va unido al de lujo, precio elevado. Un servicio de calidad debe responder al valor que el cliente está dispuesto a pagar. En cambio por otro lado, Vaquero (2013) menciona que la finalidad de la calidad de servicio es dar lo que el consumidor y/o cliente espera recibir por un producto o servicio, sólo de esta manera el cliente estará satisfecho con lo que ha consumido.

En la calidad del servicio influye tanto la percepción del cliente como la expectativa, sobre este último Menorca, Ayensa \& Borondo (2014) señalaron que las expectativas son como una evaluación mental que realiza el individuo del servicio que le van a prestar. En las expectativas se analiza el enfoque de comparación, el enfoque de cantidad ideal y el enfoque de niveles.

En tanto que para Gallwey (2013) las expectativas son el resultado de esperar con impaciencia un acontecimiento que está a punto de producirse, es diferente de la palabra esperanza en dos sentidos. Esperanza es un sentimiento que surge del deseo y no requiere ninguna razón para creer que lo que esperamos vaya realmente a ocurrir, mientras que la expectativa se basa en algunas razones que pensamos que hacen probable el acontecimiento. Mientras que Monfort, Defante \& Lima (2013) enfatizan que las expectativas pueden influenciar directamente la satisfacción gracias a la capacidad inminente de sobrestimar, colorear o sesgar la interpretación de la experiencia de consumo, confirmando o no los valores obtenidos con la compra. 
Debido a que esta investigación se refiere al área de restauración diremos que hoy en día, el buen servicio en la restauración ya no es un agregado que le otorgue una ventaja competitiva, sino es lo mínimo que debe hacer un establecimiento para entrar a competir en un mercado cada vez más exigente (Bowden-Everson \& Otros, 2013)

Para el sector de la restauración, es prioritario avanzar de acuerdo a la tendencia del mercado en el que se requiere tener la capacidad para instaurar una gestión integral y más comprometida con el cliente, haciendo énfasis en lo planteado por Gómez \& Otros (2003) redoblando esfuerzos en cuanto a eficiencia y eficacia, atención y competencia, para lograr manifestado por Monserrat (2012) que es una oferta atractiva, competitiva y variada.

En todo proceso es importante considerar la etiqueta y el protocolo, lo que implica la aplicación de normas de comportamientos establecidos para hacer la vida social más agradable, pero la diferencia se marca en que la etiqueta regula la conducta personal y el protocolo establece los criterios de trato y procedencia de una persona o institución, para Pérez \& Gardey (2009), la etiqueta no siempre implica reglas protocolarias, en cambio el protocolo sí incluye reglas de etiqueta en el comportamiento de cada persona. En síntesis, es un actuar que engloban el comportamiento de profesionales de ciertos campos.

Para asegurar la calidad del servicio, es necesario evaluarla desde una perspectiva de las expectativas, que parte de la necesidad de atender los requerimientos del cliente, lo que supone incluir factores subjetivos relacionados con los juicios de las personas que reciben el producto o servicio (Morales \& Hernández, 2004). Al ser la calidad un proceso integrado compuesto por varios elementos - Expetativas vs. Percepciones, Parasuraman et al. (1985, 1988), citados por Velásquez (2011), partieron de la conceptualización de Gronroos para desarrollar un instrumento de medida de la calidad de servicio percibida: SERVQUAL, quienes, a partir de un extenso grupo de entrevistas, identificaron diez determinantes de la calidad de servicio: 1. Elementos tangibles. Apariencia de las instalaciones físicas, equipos, personal y materiales de comunicación. 2. Fiabilidad. Habilidad para ejecutar el servicio prometido de forma fiable y cuidadosa. 3. Capacidad de respuesta. Disposición para ayudar a los clientes, y para proveerlos de un servicio rápido. 4. Profesionalidad. Posesión de las destrezas requeridas y conocimiento del proceso de prestación del servicio. 5. Cortesía. Atención, consideración, respeto y amabilidad del personal de contacto. 6. Credibilidad. Veracidad, creencia, y honestidad en el servicio que se provee. 7. Seguridad. Inexistencia de peligros, riesgos o dudas. 8. Accesibilidad. Lo accesible y fácil de contactar. 9. Comunicación. Mantener a los clientes informados, utilizando un lenguaje que puedan entender, así como escucharles. 10. Compresión del cliente. Hacer el esfuerzo de conocer a los clientes y sus necesidades.

De esta forma, el modelo SERVQUAL de calidad de Servicio permite disponer de puntuaciones sobre percepción y expectativas respecto a cada característica del servicio evaluado. La diferencia entre percepción y expectativas indicará los déficits de calidad cuando la puntuación de expectativas supere a la percepción (Mezzoni, 2015). 


\section{Métodos}

En la investigación, se aplicó el instrumento modelo de evaluación SERVQUAL que proporciona información detallada sobre; opiniones del cliente sobre el servicio de las empresas, comentarios y sugerencias de los clientes de mejoras en ciertos factores, impresiones de los empleados con respecto a la expectativa y percepción de los clientes (Matsumoto, 2014), para mejorar la calidad del servicio en el restaurante TAPAS \& TOPES de la ciudad de Puyo, bajo esta estructura, se levantaron datos requeridos en el estudio sobre cinco dimensiones: Elementos tangibles, confiabilidad, capacidad de respuesta, seguridad y empatía.

Cuadro 1: Dimensiones y significado de la calidad del servicio

\begin{tabular}{l|l}
\hline \multicolumn{1}{c|}{ DIMENSIÓN } & \multicolumn{1}{c}{ SIGNIFICADO } \\
\hline SEGURIDAD & $\begin{array}{l}\text { Conocimiento del servicio prestado por los } \\
\text { empleados, así como su habilidad para } \\
\text { transmitir confianza al cliente e información. } \\
\text { Apariencia de las instalaciones físicas, equipos, } \\
\text { empleados y facilidades. } \\
\text { Habilidad de prestar el servicio ofertado de } \\
\text { forma precisa y confiable, conforme a un } \\
\text { horario establecido según el servicio solicitado. } \\
\text { Disposición y voluntad para ayudar a los } \\
\text { clientes, el servicio oportuno para resolver las } \\
\text { demandas de un cliente. } \\
\text { Cortesía, atención personalizada que ofrece el } \\
\text { restaurante a sus clientes. }\end{array}$ \\
\hline EMPATÍA
\end{tabular}

Para identificar las áreas a ser evaluadas, se realizó un diagnóstico del servicio que brinda el restaurante y dentro de ello se establecieron 32 ítems, que están asociados a los indicadores de calidad del servicio. La escala utilizada fue tipo Likert de cinco categorías de respuesta, (5) Excelente; (4) Muy Buena (4); (3) Buena; (2) Deficiente y (1) Muy deficiente. El cuestionario consta de treinta y dos (32) ítems enfocados a factores de expectativa y percepción de la calidad del servicio por parte de los clientes que acuden al restaurante.

Cuadro 2: Variables de la calidad del servicio.

\begin{tabular}{|c|c|c|}
\hline \multicolumn{2}{|r|}{ VARIABLES } & \multirow{2}{*}{ DIMENSIÓN } \\
\hline 1.- $S 1$ & RVICIO EN LAS INSTALACIONES & \\
\hline 1.1 & $\begin{array}{l}\text { Cuidan del aspecto interno y externo del establecimiento (color, decoración, } \\
\text { diseño) }\end{array}$ & Elemento tangible \\
\hline 1.2 & $\begin{array}{l}\text { El entorno audiovisual e iluminación son adecuados para el tipo de } \\
\text { restaurante }\end{array}$ & Elemento tangible \\
\hline
\end{tabular}


1.3 Existe comodidad, el mobiliario y los espacios son confortables

1.4 Cuida de la higiene, limpieza de las instalaciones, vajilla y utensilios

1.5 El restaurante cuenta con máquinas y equipos de apariencia moderna

1.6 Se ofrece información sobre las normas de comportamiento en las instalaciones del restaurante

2.- SERVICIO EN ACCESIBILIDAD

2.1 El establecimiento brinda las facilidades para acomodar el vehículo de forma segura

2.2 Existe facilidades y alternativas de pago sea en efectivo, tarjeta, etc.

2.3 El personal de recepción recibe al cliente con cortesía

2.4 El restaurante cumple con los horarios establecidos

2.5 El restaurante informa oportunamente cuando no prestará sus servicios y el por qué

2.6 El restaurante ofrece el servicio en horarios convenientes a las necesidades del cliente

\section{3.- SERVICIO DEL PERSONAL}

3.1 El personal del restaurante tiene apariencia adecuada y pulcra

3.2 El personal atiende con rapidez las demandas del cliente

3.3 Percepción acerca del trato hacia el cliente reflejado en entendimiento de necesidades, amabilidad y respeto

3.4 Frente alguna queja y/o problema, el personal del restaurante ofreció solucionarlo y estuvo dispuesto ayudar.

3.5 El comportamiento del personal inspira confianza en la entrega del servicio

3.6 Ofreció información detalla del menú diario con sugerencias de algún plato en especial

3.7 El personal se muestra perceptivo ante inquietudes y sugerencias del cliente y tiene el conocimiento para responder preguntas.

\section{4.- SERVICIO DE COMIDA}

4.1 La comida que brinda el restaurante luce apetitosa

4.2 El restaurante le ofrece un servicio rápido

4.3 El restaurante comprende las necesidades alimenticias del cliente

4.4 El sabor y olor de los alimentos que se sirvió tienen un sabor agradable

4.5 El restaurante tiene variedad en el menú con opciones para elegir

4.6 La presentación de los alimentos son visualmente atractivos

4.7 Los alimentos se perciben limpios, desinfectados y con una apariencia de estar en buen estado con propiedades naturales

4.8 Los alimentos están a la temperatura adecuada los calientes se sirve caliente y lo frío se sirve frío.

4.9 El cliente recibe la misma calidad del servicio de comida bajo cualquier tipo de circunstancia

\section{5.- OTROS SERVICIOS}

5.1 El cliente percibe que el restaurante tiene cierto grado de reconocimiento

5.2 El cliente percibe que lo solicitado en el servicio se le entregará según lo que ofrece el restaurante.

6.- CALIDAD DEL SERVICIO EN GENERAL DEL CLIENTE

6.1 El nivel de satisfacción después que hace uso del servicio del restaurante

6.2 En general el servicio prestado por el restaurante cumple con sus expectativas Elaborado: Autoras
Elemento tangible

Elemento tangible

Elemento tangible

Seguridad

Elemento tangible

Confiabilidad

Empatía

Confiabilidad

Confiabilidad

Empatía

Elemento tangible

Capacidad de respuesta

Seguridad

Confiabilidad

Seguridad

Seguridad

Empatía

Confiabilidad

Capacidad de respuesta

Empatía

Elemento tangible

Capacidad de respuesta

Elemento tangible

Elemento tangible

Capacidad de respuesta

Confiabilidad

Seguridad

Confiabilidad

Confiabilidad

Seguridad 
Para medir o evaluar la aplicación de normas de etiqueta y protocolo en el restaurante, se consideró la técnica de estudio Observación directa y el instrumento utilizado es el Check lists o lista de verificación con escala numérica, que consisten en un repertorio de cualidades, conductas o comportamientos, a los que subyace una determinada competencia, sobre los cuales el evaluador debe constatar su presencia o ausencia de conocimientos en el evaluado (Flores, 2007). La ventaja de los checklist es que, además de sistematizar las actividades a realizar, una vez rellenados sirven como registro, que podrá ser revisado posteriormente para tener constancia de las actividades que se realizaron en un momento dado.

Cuadro 3: Lista de verificación aplicación de normas de etiqueta y Protocolo

\begin{tabular}{|c|c|c|c|c|}
\hline ITEMS EVALUADOS / PROTOCOLO DEL SERVICIO & $\mathbf{1}$ & 2 & 3 & 4 \\
\hline Realiza bien el montaje de la mesa desde la primera vez & & & & \\
\hline Recibe al cliente, se ubica a un metro de distancia de la mesa asignada & & & & \\
\hline Saluda al cliente con una cordial bienvenida y se presenta & & & & \\
\hline $\begin{array}{l}\text { Realiza una correcta acomodación en la mesa primero niños, adultos mayores, } \\
\text { damas y caballeros }\end{array}$ & & & & \\
\hline Ofrece la bebida al cliente una vez que entrega el menú o carta & & & & \\
\hline $\begin{array}{l}\text { Antes de servir la bebida revisa que la cristalería esté en buenas condiciones y } \\
\text { limpia }\end{array}$ & & & & \\
\hline $\begin{array}{l}\text { Verifica que la bebida este servida } 2 \text { centímetros abajo del borde de la } \\
\text { cristalería }\end{array}$ & & & & \\
\hline Anuncia al cliente que servirá la bebida y lo hace por el lado derecho & & & & \\
\hline $\begin{array}{l}\text { Ubica los complementos en la mesa (canasta de pan y mantequilla / otro) por } \\
\text { el lado derecho }\end{array}$ & & & & \\
\hline $\begin{array}{l}\text { Antes de servir revisa que los platos tengan su respectivo cover en perfectas } \\
\text { condiciones }\end{array}$ & & & & \\
\hline $\begin{array}{l}\text { Verifica que la presentación de los alimentos sea la adecuada según el menú y } \\
\text { lo solicitado por el cliente. }\end{array}$ & & & & \\
\hline $\begin{array}{l}\text { Sirve el plato (entrada, fuerte y postre) a cada mesa por el lado derecho y con } \\
\text { la mano derecha, diciendo al cliente: "Con permiso, menciona el menú que } \\
\text { sirve y buen provecho" }\end{array}$ & & & & \\
\hline Consulta a los clientes si necesitan algo adicional a su pedido & & & & \\
\hline $\begin{array}{l}\text { Retira por el lado derecho y con la mano derecha los platos diciendo al cliente: } \\
\text { "Con permiso Sr.(a), me permite retirar?" }\end{array}$ & & & & \\
\hline $\begin{array}{l}\text { Retira el plato del postre por el lado izquierdo y por el derecho los cubiertos } \\
\text { y/o tazas, centro de azúcar, evitando hacer ruido con el manejo de la cubertería }\end{array}$ & & & & \\
\hline $\begin{array}{l}\text { Despide al cliente con un agradecimiento y una invitación a visitar } \\
\text { nuevamente el restaurante }\end{array}$ & & & & \\
\hline $\begin{array}{l}\text { Limpia la mesa y deja en la mesa la copa para agua y la servilleta, porque estas } \\
\text { se retiran al momento de desmontar la mesa. }\end{array}$ & & & & \\
\hline
\end{tabular}


Elaborado: Autoras

Cuadro 4: Rango o Categorías

\begin{tabular}{|l|l|}
\hline \multicolumn{2}{|c|}{ RANGOS O CATEGORÍAS } \\
\hline 1 & Nada / Desconoce totalmente \\
\hline 2 & Poco / Necesita capacitación o inducción \\
\hline 3 & Suficiente / Maneja normas básicas \\
\hline 4 & Mucho / Alto grado de conocimiento dominado \\
\hline
\end{tabular}

Elaborado: Autoras

\section{Resultados y discusión}

Mediante la técnica conocida como sondeo, que su objetivo es aportar una primera impresión y descripción global de la realidad de un objeto de estudio de la zona seleccionada como área de trabajo (Serna, como se citó en Pineros, 2009), se registró que el promedio de clientes que consumen en el restaurante por semana (de lunes a sábado) es de 145, que al mes serían 580 clientes considerados como el universo o la población, se calculó la muestra siendo el resultado $n=232$ clientes consultados, a quienes se aplicó, una encuesta estructura en un periodo de tres semanas establecidas para la recolección de información.

Las variables de la calidad del servicio en conjunto explican a la opinión general de la calidad del servicio, si realizamos un análisis por dimensión, se denota que un porcentaje entre el $85 \%$ y $95 \%$ opina que el servicio que presta el restaurante TAPAS \& TOPES es de Muy buena calidad, sobresale aquellos factores que son parte de los elementos tangibles, tal como se observa a continuación:

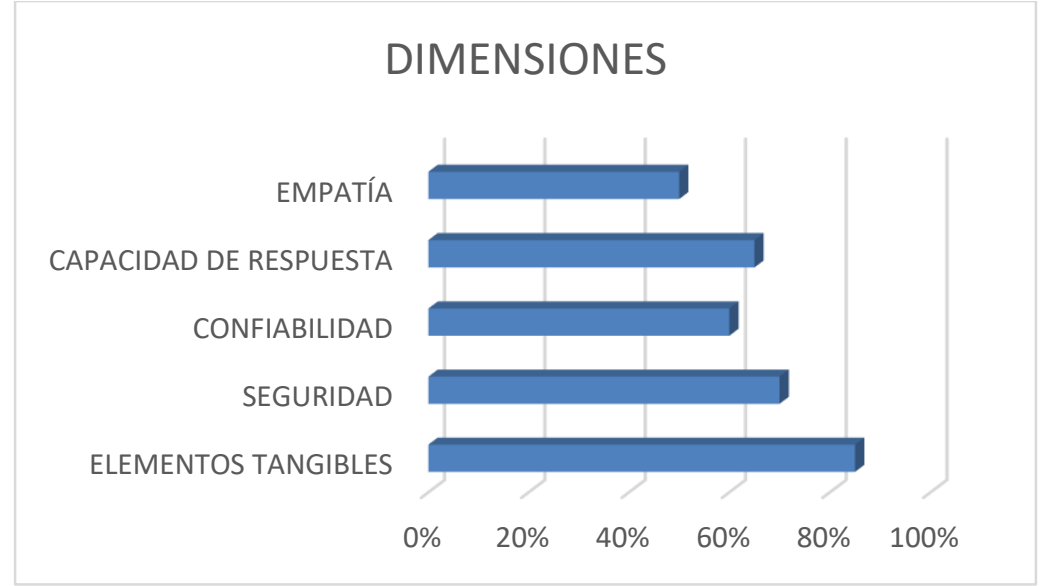

Figura 1: Dimensiones de la calidad del Servicio

Elaborado en base a datos recolectados encuesta. 
Los valores que se obtienen por rango de todos los ítems señalados, según la opinión de los clientes frente a la calidad de cada factor son: 6,2\% Excelente; 59,37 \% Muy Buena; 28,1\% Buena; 6,25\% Deficiente y 0\% Muy deficiente

Dentro de los elementos tangibles el ítem 1 "Cuidado del aspecto interno y externo del establecimiento", fue calificado por un $90 \%$ de los clientes como Excelente, mientras que el ítem 6 "El establecimiento brinda las facilidades para acomodar el vehículo de forma segura", fue calificado por un $87 \%$ como deficiente. Es importante considerar que de los 10 factores correspondientes a esta dimensión 8 fueron valorados con calidad de Buena y Muy Buena por los clientes, lo cual se constituye una fortaleza para el establecimiento que no debe descuidar elementos que son visibles y apreciados por el cliente.



Figura 2: Dimensión Elementos Tangibles

Elaborado en base a datos recolectados encuesta

Las empresas turísticas son empresas de servicios y, si bien prestan servicios no asociados a la adquisición de un bien tangible, para ello necesitan contar con importantes infraestructuras y equipamientos convirtiéndose así en grandes consumidoras de productos industriales, tal es el caso de los restaurantes que para montar un negocio de este nivel no solo se trata de saber cocinar muy bien, sino de tener conocimientos claves en gestión, organización, finanzas, etc., para que el negocio perdure en el tiempo y pueda enfrentar los problemas propios de crecimiento y desarrollo económico de su establecimiento, que debe cuidar cada detalle relacionado a la calidad del servicio para conseguir la satisfacción del cliente y alcanzar su fidelización con el producto.

El modelo SERVQUAL, define la seguridad como el conocimiento y atención mostrados por los empleados y sus habilidades para inspirar credibilidad y confianza, en la encuesta aplicada a los clientes, se consideró 6 ítems, siendo el más factor del ítem 6 "En general el servicio prestado por el restaurante cumple con sus expectativas", el más valorado por los clientes con un $88 \%$, en tanto que el ítem 1 "Se ofrece información sobre las normas de 
comportamiento en las instalaciones del restaurante", los clientes en un $90 \%$ valoraron como Deficiente.

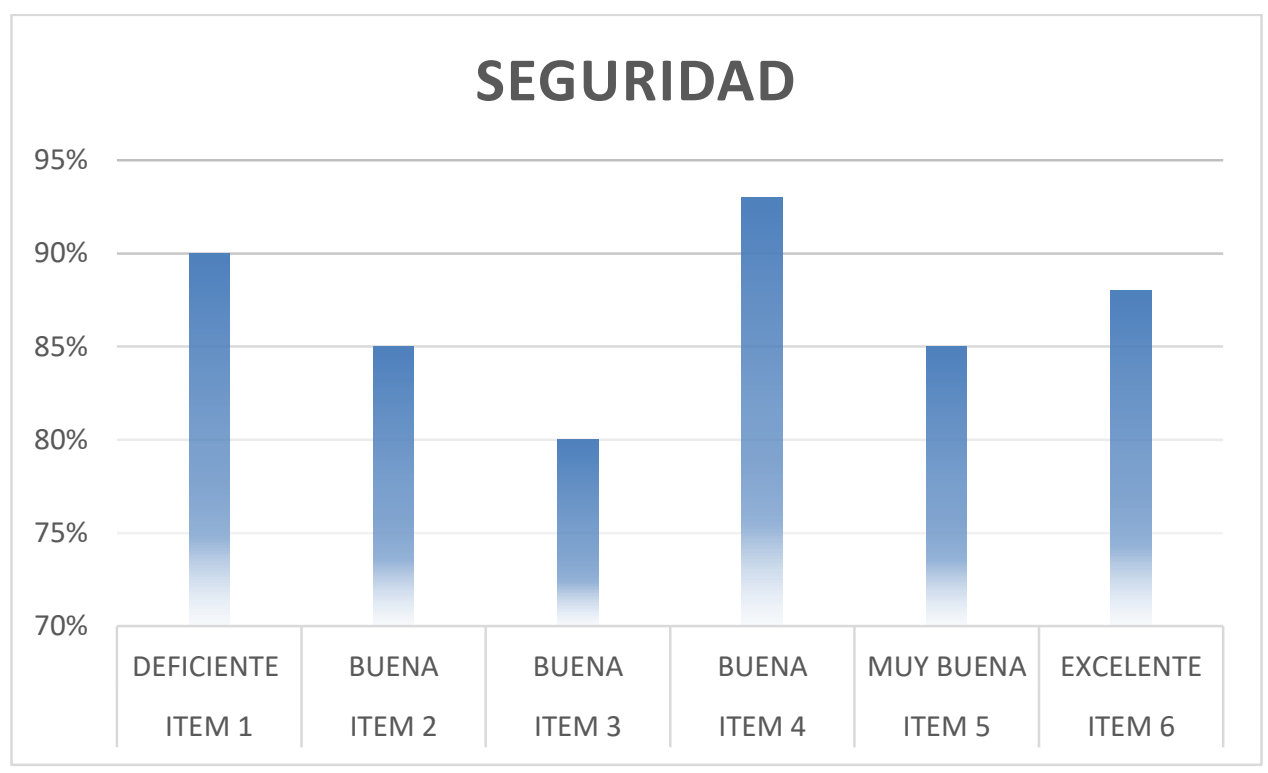

Figura 3: Dimensión Seguridad

Elaborado en base a datos recolectados encuesta

Cuando un cliente contrata un servicio, a diferencia de cuando compra un producto, no está viendo con sus ojos lo que va a recibir, si bien pudo haber recibido información previa, hasta que no consume el servicio, no podrá evaluar si es lo que necesitaba. Por este motivo es tan importante dar al servicio una de sus dimensiones clave: SEGURIDAD. Si el cliente no percibe que la empresa sabe lo que está haciendo, temerá no recibir un buen servicio y acudirá a otra empresa que le inspire esa seguridad. En el caso del restaurante TAPAS \& TOPES, es necesario que se publicite con un rótulo al ingreso que informe al cliente sobre las normas de comportamiento dentro del establecimiento para que de manera oportuna sobre todo el cliente que acude con niños tome las precauciones del caso.

Una parte del buen servicio que se debe brindar al cliente es la capacidad de respuesta, en esta dimensión al aplicar la encuesta a los clientes del restaurante sobre los 4 ítems considerados, todos fueron calificados en el rango de Bueno a Muy Bueno, siendo el ítem 3 "El restaurante tiene variedad en el menú con opciones para elegir", el que recibió el mayor porcentaje de valoración del cliente que fue de $93 \%$. 




Figura 4: Dimensión Capacidad de Respuesta

Elaborado en base a datos recolectados encuesta

Mantener contentos a los clientes es la clave para asegurar que los clientes actuales no se conviertan en clientes pasados. El no poder cumplir con la entrega del servicio de forma oportuna o hacer caso omiso a las demandas del cliente, puede aumentar su insatisfacción con tu empresa y estimularlos a investigar los servicios de la competencia.

El restaurante TAPAS \& TOPES ha dado prioridad a la capacidad de respuesta al cliente porque es aquí donde ha encontrado las oportunidades de servir a tus clientes, para que los problemas que pueden presentarse en el proceso de servicio de comida disminuyan. Esta dimensión refleja el compromiso del restaurante en brindar un servicio de calidad, que es organizado en tiempos y espacio, aun cuando este último aspecto que es el espacio no es tan amplio como en el caso de otros establecimientos que tiene el mismo servicio gourmet.

Para alcanzar expectativas positivas ante el cliente es importante ser cuidadoso con la Confiabilidad, para ejecutar el servicio prometido de forma fiable. En esta dimensión se consideraron 8 ítems o preguntas que se plantearon al cliente y todas sus respuestas se ubicaron en el rango de Bueno a Muy Bueno. El que mayor valoración alcanzó por parte del cliente fue el ítem 4 "Frente algún aqueja y/o problema, el personal del restaurante ofreció solucionarlo y estuvo dispuesto ayudar”, con una calificación de Muy Buena para un 85\%, seguida por el ítem 7 "El cliente percibe que lo solicitado en el servicio se le entregará según lo que ofrece el restaurante", con un 80\% de calificación Muy Buena. 


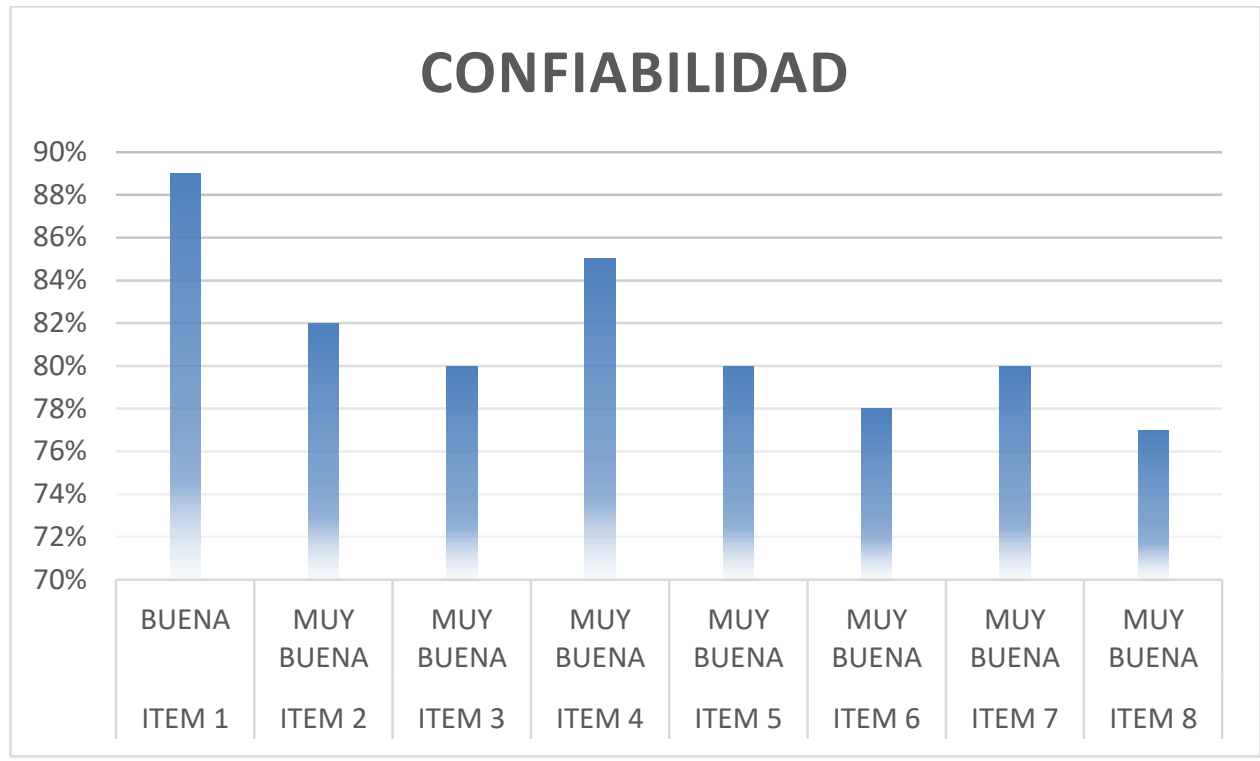

Figura 5: Dimensión Confiabilidad

Elaborado en base a datos recolectados encuesta

La confiabilidad de un servicio contribuye fuertemente a la imagen de marca y es considerada por la mayoría de los clientes como una dimensión fundamental de la calidad de un servicio, porque en ella los clientes depositan su confianza sobre el establecimiento, por lo tanto, es vital asegurarse primero que el servicio ofrecido sea fiable para publicitarlo al cliente porque está en juego la primera impresión que se lleva del servicio, porque la fiabilidad está estrechamente relacionada con el desempeño.

La empatía nos permite establecer vínculos más sólidos y positivos con los demás, dentro del servicio al cliente, se define como la capacidad para reconocer y entender las circunstancias que les pueden afectar en situaciones concretas cuando se está recibiendo el servicio. Para evaluar la calidad del servicio en esta dimensión se consideró 4 ítems, de los cuales el que más alta valoración alcanzó, en el rango de Buena a Muy Buena, es el ítem 1 "El personal recibe al cliente con cortesía", con un total de $95 \%$ de clientes que calificaron a este comportamiento con Muy Buena, en tanto que al ítem 3 "El restaurante comprende las necesidades alimenticias del cliente", recibió una calificación de Buena en un 90\%. 




Figura 6: Dimensión Empatía

Elaborado en base a datos recolectados encuesta

Tratar a los clientes como personas, mediante atención individualizada y cuidadosa, permite transmitir el mensaje que son únicos y especiales. En este tipo de negocios como un restaurante con frecuencia el mesero está en contacto con el cliente e incluso puede conocer su nombre. Este aspecto debe ser considerado como una estrategia competitiva que el establecimiento debe instaurar como política, ya que una respuesta con palabras adecuadas y de cortesía impactan al cliente quién determinará el retorno y tomará la decisión de consumir el producto solo por el buen trato que recibe del establecimiento.

En la medición sobre las normas de etiqueta y protocolo del personal que labora en el restaurante Tapas \& Topes que son 2 meseros, al aplicar el check list o lista de verificación, se puede observar que de los 17 ítems evaluados el de mayor porcentaje que corresponde al $41 \%$ se encuentran en el rango de "Poco conocimiento", es decir el personal en el cumplimiento de este proceso del servicio, necesitan de capacitación o inducción, para mejorar en aspectos fundamentales que son parte de la atención que se brinda al cliente diariamente. 


\section{EVALUACIÓN NORMAS DE PROTOCOLO \& ETIQUETA - SERVICIO RESTAURANTE TAPAS\&TOPES}

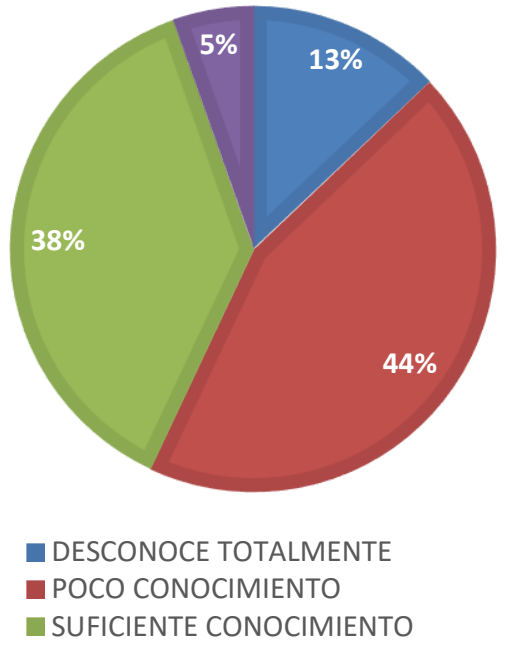

Figura 7: Evaluación normas de protocolo y etiqueta

Elaborado en base a datos recolectados encuesta

Es importante recordar que Protocolo y etiqueta son dos valores que no pasan de moda en un restaurante. La forma en que los clientes son atendidos es parte de la vivencia de los comensales, de su experiencia en el almuerzo o la cena. El tratamiento que reciben del personal y el respeto a las normas de servicio de mesa, son considerados como factores que incidirán en las ganas de volver. Aunque el estilo del local sea informal, existen normas que siempre deben tomarse en cuenta, una cosa es la decoración y el menú y otra el comportamiento del mesero, quién con el protocolo y etiqueta determinarán los pasos y acciones a seguir para atender a los clientes y satisfacer sus demandas.

\section{Conclusiones.}

- Con respecto a las variables de la calidad del servicio, tanto tangibles como intangibles, estas tienen un impacto importante sobre la expectativa y percepción del cliente. Entonces, si un cliente recibe un buen servicio es natural que le despierte una actitud favorable hacia el restaurante, traduciéndose en una cierta intencionalidad de repetir nuevamente la experiencia, aunque otros restaurantes pueden estar generando inclinaciones similares. Adicionalmente, es difícil pensar que un cliente de restaurante desee elegir siempre una misma opción de consumo, por mucho que le guste el restaurante, debiendo intervenir aquí aspectos de búsqueda de diversidad.

- Por otra parte, resultó muy provechos aplicar el modelo SERVQUAL, porque nos indica la línea a seguir para mejorar la calidad de un servicio, aportando 
fundamentalmente, en reducir determinadas discrepancias. En primer lugar, se asume la existencia de cinco deficiencias en el servicio. Cada una asociada a un tipo de discrepancia. Los elementos tangibles del restaurante deben ser bien aprovechados porque ante la percepción del cliente son los que más valor tienen para medir la calidad en este caso en particular, también está la Confiabilidad en la cual debe trabajar el establecimiento elevando aquellos factores que no superaron el rango de calificación "Bueno".

- Si bien no se encontraron relaciones sólidas entre las variables individuales de la calidad del servicio hacia la satisfacción del cliente, sí se encontraron relaciones considerables entre éstas y la opinión general. Los resultados obtenidos pueden ser útiles a los administradores de restaurantes para identificar áreas en las que se podría poner mayor atención, tanto al diseñar estrategias como en el funcionamiento diario del restaurante, logrando mayor influencia en una actitud favorable por parte del cliente (opinión general) favoreciendo ligeramente su intención de regresar a comer (lealtad intención). Estas áreas de servicio en las cuales se sugiere poner mayor atención implican aspectos como calidad y abundancia de los alimentos, aspecto del restaurante, atención personalizada y homogeneidad en la prestación del servicio (que siempre cumpla).

- Para futuras investigaciones sería interesante incluir como variables dependientes de la lealtad planteadas por Oliver (1999): lealtad cognitiva, afectiva, intención y acción.

- Además, se sugiere estandarizar las normas de Protocolo y Etiqueta como parte del servicio y factor de calidad que puede incluirse en una de las dimensiones consideradas en la herramienta de evaluación. A pesar que generalmente, cada restaurante adapta el protocolo estándar a sus necesidades y todos los meseros deben cumplirlo.

\section{Referencias bibliográficas.}

Bowden-Everson, J. Dagger, T. \& Elliot, G. (2013). Engaging Customers for Loyalty in the Restaurant Industry: The Role of Satisfaction, Trust, and Delight. Journal of Foodservice Business Research, 16 (1), 52-75.

Cantú, H. (2011). Desarrollo de una cultura de calidad. México: Mc.Graw Hill.

Duque, E. (2005). Revisión del concepto de calidad del servicio y sus modelos de medición. INNOVAR, 15 (25), 17.

Flores, G. (2007). La evaluación de competencias laborales Educación XX1, núm. 10, 2007, pp.95 Universidad Nacional de Educación a Distancia Madrid, España.

Gallwey, W. (2013). El juego interior del golf. Madrid: Sirio.

Gómez, R. Medina, N. \& Escalona, M. (2003). Doosier de Gestión de Alimentos y Bebidas. Escuela de Altos Estudios de Hotelería y Turismo. La Habana, Cuba. 
Matsumoto, R. (2014). Desarrollo del Modelo Servqual para la medición de la calidad del servicio en la empresa de publicidad Ayuda Experto. Revista Perspectivas, (34), 181209. Recuperado en 06 de octubre de 2018, de http://www.scielo.org.bo/scielo.php?script=sci_arttext\&pid=S1994$37332014000200005 \& \operatorname{lng}=\mathrm{es} \& \ln \mathrm{l}=\mathrm{es}$.

Menéndez, M. \& Motto, M. (2014). Servicio y atención al cliente en restaurante. Madrid, España: Ideas propias.

Menorca, C., Ayensa, E., \& Borondo, J. (2014). La calidad en las organizaciones turísticas. Madrid, España: Paraninfo.

Monfort, M., Defante, L., Lima, D. (2013). Satisfacción del consumidor de servicios hoteleros implicaciones estrategias. Revista Estudios y Perspectivas en Turismo, pp. 276-293. Buenos Aires: Argentina.

Monserrat, G. (2012). Técnicas de Servicio de Alimentos y Bebidas en barra y mesa. Mise en placey servicio al cliente en establecimientos de restauración. Primera Edición. Editorial: Ideas propias. España.

Morales, S. \& Hernández, A. (2004). Calidad y Satisfacción en los servicios: Conceptualización. Efedeportes Revista Digital, 10(73).

Olivier, R. (1999). Whence customer loyalty. Journa of Marketing. New York. 63 (4): 1233.

Pérez, J. \& Gardey, A. (2009). Definición de insumos. Recuperado:9 de octubre 2018. Sitio web: http://definicion.de/insumo/

Pineros, J. (2009). Medición de la satisfacción del cliente del restaurante Museo Taurino. Universidad Javeriana. Bogotá, Colombia.

Vaquero, J. (2013). Servicio en restaurante. Madrid, España: CEP, S.L.

Velásquez, D. (2011). Medición de la satisfacción de usuarios de la Universidad del Valle. Cali: Univalle.

Vera, J. \& Trujillo, A. (2009). El Papel de la calidad del servicio de Restaurante como antecedente de la lealtad del cliente. Revista Panorama Socioeconómico. Vol. 27, número 38, pp. 16-30. Universidad de Talca. Chile.

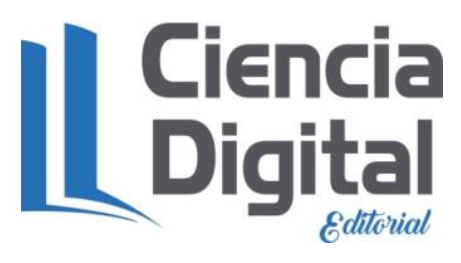




\section{Para citar el artículo indexado.}

Cevallos T., Bastidas M., Fierro A. \& Ordóñez E. (2019). Indicadores para medir la calidad del servicio y normas de protocolo y etiqueta, aplicando el modelo SERVQUAL, en el restaurante TAPAS \& TOPES, de la ciudad de Puyo, provincia de Pastaza. Revista electrónica Ciencia Digital 3(1), 75-91. Recuperado desde: http://cienciadigital.org/revistacienciadigital2/index.php/CienciaDigital/article/view/263/567

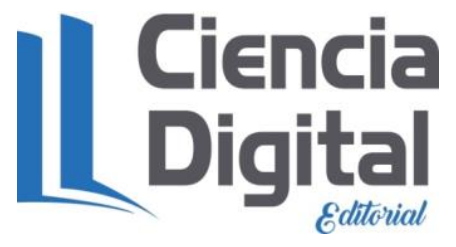

El artículo que se publica es de exclusiva responsabilidad de los autores y no necesariamente reflejan el pensamiento de la Revista Ciencia Digital.

El articulo queda en propiedad de la revista y, por tanto, su publicación parcial y/o total en otro medio tiene que ser autorizado por el director de la Revista Ciencia Digital.
